$V_{T}$

NCER Working Paper Seriés

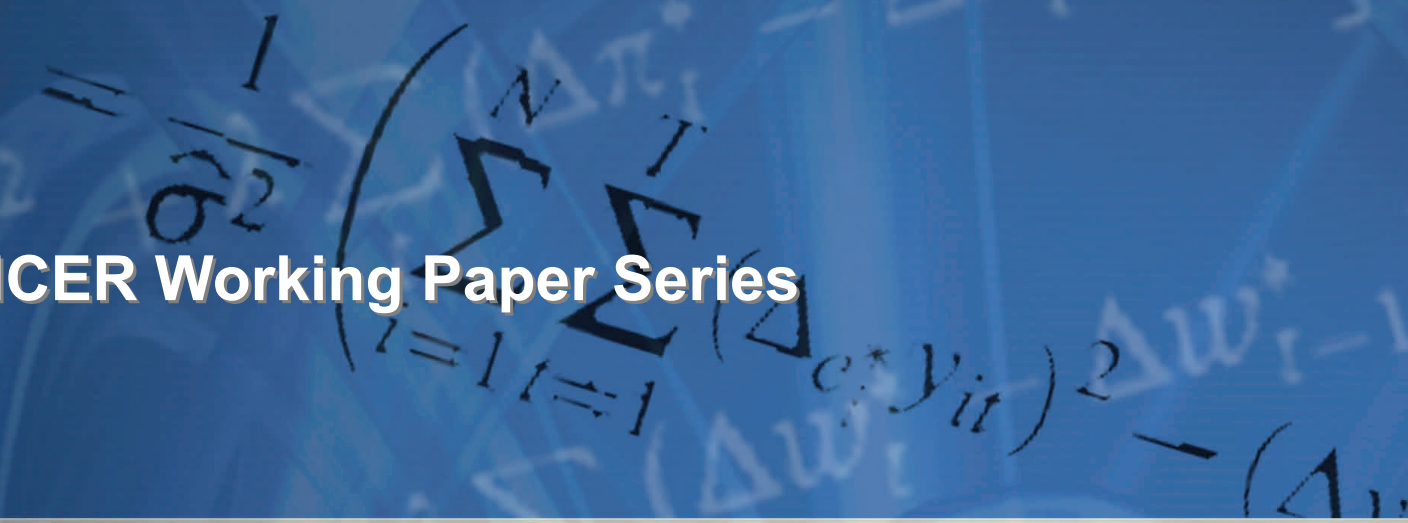

Structural Credit Risk Model with

Stochastic Volatility:

A Particle-filter Approach

Di Bu

Yîn Liao

Working Paper \#98

October 2013 


\title{
Structural Credit Risk Model with Stochastic Volatility: A Particle-filter Approach*
}

\author{
$\mathrm{Di} \mathrm{Bu}$ \\ University of Queensland \\ Brisbane, QLD, 4000 \\ AUSTRALIA
}

\author{
Yin $\operatorname{Liao}^{\dagger}$ \\ Queensland University of Technology \\ Brisbane, QLD, 4000 \\ AUSTRALIA
}

October 29, 2013

\begin{abstract}
This paper extends Merton's structural credit risk model to account for the fact that a firm's asset volatility follows a stochastic process. With the presence of stochastic volatility, the transformed-data maximum likelihood estimation (MLE) method of Duan $(1994,2000)$ can no longer be applied to estimate the model. We devise a particle filtering algorithm to solve this problem. This algorithm is based on the general non-linear and non-Gaussian filtering with sequential parameter learning, and a simulation study is conducted to ascertain its finite sample performance. Meanwhile, we implement this model on the real data of companies in Dow Jones industrial average and find that incorporating stochastic volatility into the structural model can improve the model performance significantly.
\end{abstract}

Keyword: Credit risk; Merton model; Stochastic volatility; Particle filter; Default Probability; CDS;

JEL: C22

${ }^{*}$ We thank Tom Smith and Jin-Chuan Duan for helpful comments, as well as conference participants at the 4th International Conference on Computational and Financial Econometrics, the 2013 University of Queensland Business School Research Colloquium and seminar participants at University of Queensland and Australian National University. All remaining errors are ours.

†Corresponding author. Email address: yin.liao@qut.edu.au 


\section{Introduction}

In the past few years, financial markets have been experiencing an unprecedented crisis. As a result, the academic literature on modeling credit risk has been growing fast. Currently, there are two main approaches to modeling credit risk. One is called the reduced form approach, which considers default as an exogenous event. The other one is a structural approach that was first developed in Merton (1974), and henceforth is called the Merton model. The Merton model assumes that a firm's asset value follows a geometric Brownian motion with a constant volatility, and the firm's capital structure consists of a zero-coupon debt and common equity. The default is consequently treated as an endogenous decision usually made by the firm's equity holders. However, one of the assumptions in the Merton model, that the firm's asset return has a constant volatility, has a long history of criticism. This paper presents a structural credit risk model with stochastic volatility (SV structural model). In particular, we employ the stochastic volatility model to describe the evolution of a firm's asset value, and derive the corresponding credit risk measures of the firm based on this model.

There are compelling reasons for incorporating stochastic volatility into the structural model to estimate credit risk. From a theoretical perspective, estimating the structural credit risk model largely depends on the value of the firm's equity, which is regarded as a call option written on the assets of the firm. Since volatility plays a crucial role in pricing options, it will also have a major impact on structural model estimation. At the empirical level, academics and practitioners have long noted that the volatility of a firm's assets changes through time. Some studies have shown that the constant volatility assumption is too restrictive and causes the Merton model to estimate the credit risk measures with a large bias. Jones et al. (1984) analyzed 177 bonds issued by 15 firms and found that the Merton model overestimates bond prices by $4.5 \%$ on average. Ogden (1987) shows that the Merton model underpredicts the bond yield spread by 104 basis points on average. Eom et al. (2004) empirically test the Merton model in terms of estimating credit default swap (CDS) spread and find that this model generates a very large estimation error. Tarashev (2005) suggested that the default probability generated by the Merton model is significantly less than the empirical default rate. In general, as the relationship between the firm's asset value

and its debt obligation is fundamental to corporate credit risk, a stochastic volatility model will help us more properly describe the firm's asset price evolution, and more accurately measure its credit risk exposure. 
The objectives of this paper are three-fold. First, we develop a new structural model of credit risk that explicitly accounts for stochastic volatility in a firm's asset return. Particularly, we employ the Heston model as an example to illustrate how to use this type of model in credit risk estimation. Second, we devise a transformed-data particle filtering algorithm to estimate the model, and this technique is based on the general non-linear and non-Gaussian filtering approach. In general, the stochastic volatility (SV) structural model can be regarded as a non-standard state space model with the asset value being unobserved. It is worth noting that several issues make the model estimation challenging. One is that due to the unobserved asset values many existing approaches used for estimating the standard state space model can't be directly applied. Second, when the asset return has a stochastic volatility, the likelihood function of the returns based on the observed equity prices is no longer available in a closed form. The standard MLE method can't be used to estimate the model. Lastly, the state variable that determines the level of volatility is not directly observed, which increases the dimension of the latent variables that need to be estimated. The algorithm we developed here is able to address each of these issues. We firstly employ a transformation technique developed in Duan (1994) to obtain the implied asset values from the observed equity values. Furthermore, we implement particle filter on the observed equity values to generate consecutive prediction and filtering distributions for the unobserved asset values and the latent stochastic volatility process by using a set of samplers. It turns out by using this algorithm the likelihood function of these observed equity values can be easily evaluated without any distribution assumptions, and the new model parameters as well as the distribution of the asset return volatility can be subsequently inferred. Third, we implement our model on the real data of companies in Dow Jones industrial average to find empirical support for this methodology. We estimate the SV structural model for these companies, and compare the performance of the SV structural model to the Merton model with respect to the credit risk estimation of these firms. The 5-year credit default swap (CDS) spread is used as a proxy of the real credit risk of these firms, and compared with the model-implied corporate credit spread to judge the ability of the models.

Overall, the simulation results show that the transformed-data particle filter algorithm is able to provide accurate estimates for the SV structural model. Meanwhile, our SV structural model fits the 5-year CDS spread much better than the Merton model. At the average level, the mean in-sample root mean square error (RMSE) and mean absolute error (MAE) of our model are respectively 100.45 and 113.33 basis points (bps), 
versus 115.30 and 129.67 bps for the Merton model; in the out-of-sample analysis, the SV structural model achieves a mean RMSE and MAE of 102.82 and 114.10bps, relative to 116.06 and $126.32 \mathrm{bps}$ for the Merton model. More importantly, at the individual firm level, our SV structural model has a lower in-sample and out-of-sample RMSE than the Merton model for every single firm in the sample.

The rest of the paper is organized as follows. Section 2 lays out the SV structural model and describes the transformed-data particle filter technique for estimating the model. Section 3 conducts a Monte Carlo simulation to study the finite sample performance of the transformed-data particle filter technique. Section 4 benchmarks the SV structural credit risk model against the Merton model using the 5-year CDS spread data of 27 companies in Dow Jones industrial average. Section 5 concludes.

\section{Structural credit risk model with stochastic volatil- ity}

This section consists of three parts. Section 2.1 describes the new model. Section 2.2 introduces a transformed-data particle filter algorithm to estimate the model. In Section 2.3, we discuss the model's application in the credit market.

\subsection{Model description}

As we know, Merton (1974) laid the foundation for the literature on the structure approach to credit risk modeling. We follow the general set-up of the Merton model, but relax the assumption that the firm's asset value has a constant volatility to allow it to follow a stochastic volatility process. The asset value and the asset return volatility of a firm at time $t$ are respectively defined as $S_{t}$ and $V_{t}$, and we employ the Heston stochastic volatility model to describe the joint dynamics of the asset value and its volatility as

$$
\begin{gathered}
d S_{t}=\mu S_{t} d t+\sqrt{V_{t}} S_{t} d W_{t}^{S} \\
d V_{t}=\kappa\left(\theta-V_{t}\right) d t+\xi \sqrt{V_{t}} d W_{t}^{V}
\end{gathered}
$$


where $d W_{t}^{S}$ and $d W_{t}^{V}$ are Wiener processes with correlation $\rho$. It should be noted that the Heston model is used as an example of the stochastic volatility model, and the rest of the analysis in this paper can be generalized to other stochastic volatility models.

Assuming that the firm has two types of outstanding claims, that is, an equity and a zero-coupon debt maturing at time $T$ with face value $F$, the following accounting identity should hold for every time $t$ as

$$
S_{t}=E_{t}+B_{t}
$$

where $E_{t}$ and $B_{t}$ are respectively the market value of equity and the market value of debt at time $t$. When debt matures, the default occurs in the event that the firm's assets fall below the face value of the debt, i.e. $S_{T}<F$. Otherwise, equity holders repay the debt and keep the balance. Therefore, the payout to the debt holders at the maturity time $T$ is

$$
B_{T}=\min \left(S_{T}, F\right)
$$

and the equity holders, on the other hand, receive

$$
E_{T}=\max \left(S_{T}-F, 0\right)
$$

at time $T$. Accordingly, the firm's equity can be regarded as if it were a call option on the total asset value of the firm with the strike price of $F$ and the maturity date $T$. Assuming the risk-free interest rate is $r$, the equity claim consequently can be priced at any time $t<T$ based on the Heston call option pricing formula ${ }^{1}$ as

$$
E_{t}=f\left(S_{t} ; V_{t}, F, r, T-t\right)=S_{t} P_{1}-F e^{-r(T-t)} P_{2}
$$

where

$$
P_{j}=\frac{1}{2}+\frac{1}{\pi} \int_{0}^{\infty} \operatorname{Re}\left(\frac{e^{-i \phi \ln (K)} f_{j}\left(x, V_{t}, T, \phi\right)}{i \phi}\right) d \phi
$$

\footnotetext{
${ }^{1}$ For other stochastic volatility models, we need to find the corresponding option pricing formula in order to do the same analysis.
} 
and

$$
\begin{gathered}
x=\ln \left(S_{t}\right), \\
f_{j}=\exp \left\{C(T-t ; \phi)+D(T-t ; \phi) V_{t}+i \phi x\right\}, \\
C=r \phi i r+\frac{a}{\xi^{2}}\left[\left(b_{j}-\rho \xi \phi i+d\right) \tau-2 \ln \left(\frac{1-g e^{d r}}{1-g}\right)\right], \\
D=\frac{b_{j}-\rho \xi \phi i+d}{\xi^{2}}\left(\frac{1-e^{d r}}{1-g e^{d r}}\right), \\
g=\frac{b_{j}-\rho \xi \phi i+d}{b_{j}-\rho \xi \phi i-d}, \\
d=\sqrt{\left(\rho \xi \phi i-b_{j}\right)^{2}-\xi^{2}\left(2 u_{j} \phi i-\phi^{2}\right)},
\end{gathered}
$$

where $j=1,2$, and we have $u_{1}=\frac{1}{2}, u_{2}=-\frac{1}{2}, a=\kappa \theta, b_{1}=\kappa+\kappa \xi V_{t}-\rho \xi$, and $b_{2}=\kappa+\kappa \xi V_{t}$. Similarly, the pricing formula for the firm's debt can be derived by regarding the payoff of the debt as the difference between a default-free debt and a put option on the total asset value of the firm with the strike price of $F$ and the maturity date $T$. This will be further discussed in Section 2.3.

\subsection{Model estimation}

To estimate this model, we regard it as a nonlinear and non-Gaussian state-space model with equation (1) being the measurement equation, and equation (2) being the latent state equation. However, two issues make the model estimation complicated.

Firstly, for an exchange listed firm, the asset values $S_{t}$ are unobservable. One can only observe a time series of equity prices instead. Therefore, although a number of estimation methods for stochastic volatility models are existing in the literature ${ }^{2}$, they can not be directly implemented on our model without transforming the equity prices into the asset values. Secondly, although this model can be regarded as a state-space model, it is nonlinear and non-Gaussian. The commonly used linear filtering technique (i.e., the standard Kalman filter) is not able to be used for solving the current problem. Even nonlinear Kalman filters, such as the extended Kalman filter and the unscented Kalman filter, will perform poorly.

Therefore, we develop a transformed-data particle filter that uses the information of the observed equity prices to estimate the parameters and the latent stochastic volatility process of this new model. Let $D_{T}$ denote a time series of the historical equity values until time $T$, i.e., $D_{T}=\left\{E_{1}, \ldots, E_{T}\right\}, \Theta$ denote the parameter vector of the model containing five parameters, i.e., $\Theta=\{\mu, \kappa, \theta, \xi, \rho\}$, and $x$ denote the latent state variable, that is, the stochastic volatility process $V_{t}$. Our objective is to estimate the parameter vector $\Theta$ and the latent state variable $x$ by using the information set $D_{T}$

\footnotetext{
${ }^{2}$ See Broto and Ruiz (2004) for a review of the estimation methods for Stochastic Volatility models.
} 
- To simplify the technical details behind the algorithm proposed here, we start with the assumption that $\Theta$ is known to explain the procedure of estimating the latent state variable $x$. Then, we move to the situations where $\Theta$ is also unknown to discuss the procedure of jointly estimating $\Theta$ and $x$.

\subsubsection{Estimating the latent state variable when the model parameters are known}

Assuming that all the parameters of the model are known, we extend the auxiliary particle filter, which is attributed to Pitt and Shephard (1999), to estimate the latent stochastic volatility process. The particle filter is a simulation-based technique to generate consecutive prediction and filtering distributions for latent state variables in the nonlinear and non-Gaussian state-space models. The technique relies on different sets of points (particles) to represent the distribution of the unobserved state variables at different time points. Bayes' rule is repeatedly used to re-weight these particles and update the distribution estimation for the unobserved state variables when new information is available.

For our particular problem, starting with the prior distribution of the latent state variable $p\left(x_{t} \mid D_{t}, \Theta\right)$, which is represented by a set of $M$ particles $\left\{x_{t}^{(i)}, i=1, \ldots, M\right\}$, the empirical prediction density of $x_{t+1}$ can be predicted according to equation (2) as

$$
\hat{p}\left(x_{t+1} \mid D_{t}, \Theta\right) \propto \frac{1}{M} \sum_{i=1}^{M} p\left(x_{t+1} \mid x_{t}^{(i)}, \Theta\right) .
$$

When time evolves to $t+1$, we observe the firm's equity value at time $t+1$ as $E_{t+1}$. Then the distribution of the latent state variables can be updated (filtered) according to Bayes' rule as

$$
\hat{p}\left(x_{t+1} \mid D_{t+1}, \Theta\right) \propto p\left(E_{t+1} \mid x_{t+1}, \Theta\right) \hat{p}\left(x_{t+1} \mid D_{t}, \Theta\right) .
$$

Equations (6) and (7) provide the basis for advancing the system from time $t$ to time $t+1$. More details are as follows:

- Step 1 - Prediction: Propagate a set of particles $\left\{x_{t}^{(i)}, i=1, \ldots, M\right\}$ from the prior distribution $p\left(x_{t} \mid D_{t}, \Theta\right)$ to $\left\{\widetilde{x}_{t+1}^{(i)}, i=1, \ldots, M\right\}$ via $p\left(x_{t+1} \mid x_{t}, \Theta\right)$. This can be easily done based on equation (2). 
- Step 2 - Resample: Assign to $\widetilde{x}_{t+1}^{(i)}$ a filtering weight of

$$
\pi_{t+1}^{(i)}=\frac{\omega_{t+1}^{(i)}}{\sum_{i=1}^{M} \omega_{t+1}^{(i)}},
$$

where $\omega_{t+1}^{(i)} \propto p\left(E_{t+1} \mid \widetilde{x}_{t+1}^{(i)}, \Theta\right)$, and resample $\left\{\widetilde{x}_{t+1}^{(i)}, i=1, \ldots, M\right\}$ with weight $\pi_{t+1}^{(i)}$ to obtain $\left\{x_{t+1}^{(i)}, i=1, \ldots, M\right\}$. Then, the filtered density $p\left(x_{t+1} \mid D_{t+1}, \Theta\right)$ can be represented by the set of resampled particles $\left\{x_{t+1}^{(i)}, i=1, \ldots, M\right\}$. This step is important, as it is an effective way to avoid the decay in the particle approximation.

We refer to Liu and Chen (1998) for a careful discussion of its merits.

As the firm's asset values $S_{t}$ are not observed, but the firm's equity values are, it is worth noting that different from dealing with the standard stochastic volatility models, in our filter algorithm we use the conditional likelihood function of equity values $p\left(E_{t+1} \mid x_{t+1}, \Theta\right)$ instead of the conditional likelihood function of asset values $p\left(S_{t+1} \mid x_{t+1}, \Theta\right)$ to generate weights for resampling. We invoke the standard result of differentiable transformations ${ }^{3}$ to derive the likelihood function for the observed equity data as

$$
\begin{gathered}
p\left(E_{t+1} \mid x_{t+1}, \Theta\right)=\frac{p\left(S_{t+1} \mid x_{t+1}, \Theta\right)}{\left|\frac{\partial f\left(S_{t+1} ; V_{t+1}, F, r, T-(t+1)\right)}{\partial S_{t+1}}\right|} \\
=\frac{1}{\sqrt{2 \pi V_{t} S_{t}^{2} d t}} \exp \left(\frac{-\left(S_{t+1}-\left(S_{t}+\mu S_{t} d t\right)\right)^{2}}{2 V_{t} S_{t}^{2} d t}\right) /\left|\frac{\partial f\left(S_{t+1} ; V_{t+1}, F, r, T-(t+1)\right)}{\partial S_{t+1}}\right|,
\end{gathered}
$$

where $S_{t+1}=f^{-1}\left(E_{t+1}, V_{t+1}, F, r, T-(t+1)\right)$ and $\frac{\partial f\left(S_{t+1} ; V_{t+1}, F, r, T-(t+1)\right)}{\partial S_{t+1}}=P_{1}^{4} \cdot f^{-1}($.$) is$ the inverse function of the option pricing formula implied from the Heston model. The inversion can be easily performed numerically using, for example, a bisection search algorithm.

However, the above propagate-resample procedure has a critical drawback. When we approximate the prediction density $p\left(x_{t+1} \mid D_{t}, \Theta\right)$ in Step 1, all the particles come from the prior distribution $p\left(x_{t} \mid D_{t}, \Theta\right)$ without taking into account the knowledge of the new observation $E_{t+1}$. The resulting weights in Step 2 may be very small on many

\footnotetext{
${ }^{3}$ Differential transformation was proposed in the 1980s by Pukhov and Zhou for the analysis of electric circuits. The basic idea is that $X$ is a random variable with pdf $f_{X}(x)$, and the function $g$ is a differentiable transformation of $X$ into $Y$, that is, $y=g(x)$. Therefore, the pdf of $Y$, the transformed random variable, is given by $f_{Y}(y)=f_{X}(x)\left|\frac{d g}{d x}\right|^{-1}$.

${ }^{4} P_{1}$ is as expressed in the equation (5).
} 
particles, and the variance of importance in the weights will grow over time resulting in a poor quality algorithm. The auxiliary variable approach proposed by Pitt and Shephard (1999) is employed to solve this problem.

The basic idea is to enlarge the dimension of the state variables by incorporating an auxiliary variable $k$ denoted as the index of the particles. In each step of the algorithm, we firstly draw a sample of particle index $k$ with size $M$ according to an importance function $p\left(E_{t+1} \mid g\left(x_{t}^{(i)}\right)\right)$, where $p\left(E_{t+1} \mid g\left(x_{t}^{(i)}\right)\right)$ is the conditional likelihood function based on a best guess for $x_{t+1}$ defined by $\hat{\mu}_{t+1}=g\left(x_{t}\right)$. The $\hat{\mu}_{t+1}$ could be, for example, the expected value, the median or mode of $x_{t+1}$ conditioning on $x_{t}$. We use the expected value in our analysis. There are two main points making the auxiliary variable idea attractive: (i) the new information $E_{t+1}$ is used in the first step to resample the $M$ particles from prior density $p\left(x_{t+1} \mid D_{t}, \Theta\right)$, and (ii) due to the pre-selection, only "good" particles are used in the following steps for propagating. The above procedures are now modified as:

- Step 1 - Resample: Draw $M$ points from $\{i, i=1, \ldots, M\}$ as indexes $\{k, k=$ $1, \ldots, M\}$ with weights

$$
\omega_{t+1}^{(i)} \propto \omega_{t}^{(i)} p\left(E_{t+1} \mid g\left(x_{t}^{(i)}\right)\right)
$$

where $p\left(E_{t+1} \mid g\left(x_{t}^{(i)}\right)\right)$ is calculated by using equation (1) along with the transformation techniques introduced above. A resampled set of particles $\left\{x_{t}^{(k)}, k=\right.$ $1, \ldots, M\}$ from $\left\{x_{t}^{(i)}, i=1, \ldots, M\right\}$ are obtained according to these sampled indexes.

- Step 2 - Prediction: Propagate $\left\{x_{t}^{(k)}, k=1, \ldots, M\right\}$ to $\left\{\widetilde{x}_{t+1}^{(k)}, k=1, \ldots, M\right\}$ via $p\left(x_{t+1} \mid x_{t}, \Theta\right)$. This can be easily done using equation (2).

- Step 3 - Resample: Assign to $\widetilde{x}_{t+1}^{(k)}$ a filtering weight of

$$
\pi_{t+1}^{(k)}=\frac{\omega_{t+1}^{(k)}}{\sum_{i=1}^{M} \omega_{t+1}^{(k)}},
$$

where $\omega_{t+1}^{(k)} \propto \frac{p\left(E_{t+1} \mid \widetilde{x}_{t+1}^{(k)}\right)}{p\left(E_{t+1} \mid g\left(x_{t}^{(k)}\right)\right)}$, and resample $\left\{\widetilde{x}_{t+1}^{(k)}, k=1, \ldots, M\right\}$ with weight $\pi_{t+1}^{(k)}$ to obtain $\left\{x_{t+1}^{(k)}, k=1, \ldots, M\right\}$. Then, the filtered density $p\left(x_{t+1} \mid D_{t+1}, \Theta\right)$ can be approximated by the set of resampled particles $\left\{x_{t+1}^{(k)}, k=1, \ldots, M\right\}$. 
Next, repeat steps 1-3 until all the equity data information has been incorporated. A final posterior density approximation $p\left(x_{T} \mid D_{T}\right)$ is our estimation for the latent state variable distribution.

\subsubsection{Jointly estimating the latent state variable and parameters}

When the parameter vector $\Theta$ in the above model is unknown, the problem becomes more complicated. A natural way to estimate these parameters is to define an autoregressive dynamic for $\Theta$, and apply the particle filter algorithm stated above to the model by incorporating these parameters in an augmented state vector $\left(x_{t}, \Theta_{t}\right)$. However, the main drawback against this approach is that it leads to time-varying but not fixed parameter estimates. More precisely, these artificial dynamics can lead to posterior variances of these actually fixed parameters ${ }^{5}$ larger than the true posteriors. To correct for this overdispersion, Liu and West (2001) propose a smooth kernel density approach to approximate the posterior distribution of the parameters $p\left(\Theta_{T} \mid D_{T}\right)$. The basic idea of the approach is to use the mixture of kernel densities to generate fresh samples from the current posterior in an attempt to avoid particle decay.

We employ this method to estimate parameters for our model. Starting with the prior distribution of the parameter vector $p\left(\Theta_{t} \mid D_{t}\right)$, which is represented by a set of $M$ particles $\left\{\Theta_{t}^{(i)}, i=1, \ldots, M\right\}$, the empirical prediction density of $\Theta_{t+1}$ can be approximated by the mixture distribution

$$
\hat{p}\left(\Theta_{t+1} \mid D_{t}\right)=\sum_{i=1}^{M} \omega_{t}^{(i)} N\left(\Theta_{t+1} \mid m_{t}^{(i)}, h^{2} \Sigma_{t}\right),
$$

where $m_{t}^{(i)}=\alpha \Theta_{t}^{(i)}+(1-\alpha) \overline{\Theta_{t}}$ is the kernel location for the $i t h$ component of the mixture, with $\overline{\Theta_{t}}$ being the mean of the $M$ particles from the prior density $p\left(\Theta_{t} \mid D_{t}\right)$, $\alpha=\sqrt{1-h^{2}}$ whereas $h^{2}=1-((3 \delta-1) / 2 \delta)^{26}$, and $\Sigma_{t}$ is the variance-covariance matrix of the $M$ particles from the prior density $p\left(\Theta_{t} \mid D_{t}\right)$. By drawing $M$ points from $\hat{p}\left(\Theta_{t+1} \mid D_{t}\right)$, the empirical density of $\Theta$ in future time points can be estimated iteratively. A final posterior density approximation $p\left(\Theta_{T} \mid D_{T}\right)$ is our estimation for the distribution of the parameter vector.

It is easy to show that the variance of the mixture approximation in equation (8) is

\footnotetext{
${ }^{5}$ Assume that the parameters in this model are constants, so the time-varying parameters are not considered here.

${ }^{6} \delta$ is a discount factor in $(0,1)$, typically around $0.95-0.99$.
} 
$\Sigma_{t}$, and the mean is $\overline{\Theta_{t}}$. It turns out that the mixture of the kernel density approach is effective to avoid the overdispersion by using location shrinkage. The shrinkage pushes particles $\Theta_{t}^{(i)}$ towards their mean $\overline{\Theta_{t}}$ when approximating the posterior density of $\Theta$ for next time, and the constants $\alpha$ and $h$ measure, respectively, the extent of the shrinkage and the degree of overdispersion of the mixture.

We now return to the more general filtering problem of the posterior density $p\left(x_{t+1}, \Theta_{t+1} \mid D_{t+1}\right)$, that is to jointly estimate the parameters and the latent state variable of the above model. Given that we have a set of particles $\left\{x_{t}^{(i)}, \Theta_{t}^{(i)}, i=1, \ldots, M\right\}$ with weights $\left\{\omega_{t}^{(i)}, i=1, \ldots, M\right\}$, representing the posterior density $p\left(x_{t}, \Theta_{t} \mid D_{t}\right)$ at time $t$, we can adopt the auxiliary particle filter discussed in Section 2.2.1 for the latent state variable coupled with the mixture of kernel densities for the parameter vector to obtain the approximation for $p\left(x_{t}, \Theta_{t} \mid D_{t}\right)$. The general algorithm is summarized as follows:

- Step1 - Resample: Compute two mean points at time $t+1$ as

$$
\begin{gathered}
\mu_{t+1}^{(i)}=g\left(x_{t}^{(i)}\right)=E\left(x_{t+1} \mid x_{t}^{(i)}, \Theta_{t}^{(i)}\right), \\
m_{t}^{(i)}=\alpha \Theta_{t}^{(i)}+(1-\alpha) \overline{\Theta_{t}},
\end{gathered}
$$

and sample a set of $M$ indexes $\{k, k=1, \ldots, M\}$ from $\{i, i=1, \ldots, M\}$ with weight

$$
\omega_{t+1}^{(i)} \propto \omega_{t}^{(i)} p\left(E_{t+1} \mid \mu_{t+1}^{(i)}, m_{t}^{(i)}\right) .
$$

- Step2 - Prediction: Propagate $\left\{\Theta_{t}^{(k)}, k=1, \ldots, M\right\}$ to $\left\{\widetilde{\Theta}_{t+1}^{(k)}, k=1, \ldots, M\right\}$ by using the kernel density defined as

$$
\widetilde{\Theta}_{t+1}^{(k)} \sim N\left(. \mid m_{t}^{(k)}, h^{2} \Sigma_{t}\right)
$$

and propagate $\left\{x_{t}^{(k)}, k=1, \ldots, M\right\}$ to $\left\{\widetilde{x}_{t+1}^{(k)}, k=1, \ldots, M\right\}$ via

$$
p\left(x_{t+1} \mid x_{t}^{(k)}, \widetilde{\Theta}_{t+1}^{(k)}\right) .
$$

- Step 3 - Resample: Compute the corresponding weight as

$$
\omega_{t+1}^{(k)} \propto \frac{p\left(E_{t+1} \mid \widetilde{x}_{t+1}^{(k)}, \widetilde{\Theta}_{t+1}^{(k)}\right)}{p\left(E_{t+1} \mid \mu_{t+1}^{(k)}, m_{t}^{(k)}\right)},
$$


and resample $\left\{\widetilde{x}_{t+1}^{(k)}, k=1, \ldots, M\right\}$ and $\left\{\widetilde{\Theta}_{t+1}^{(k)}, k=1, \ldots, M\right\}$ with weight $\pi_{t+1}^{(k)}=$ $\frac{\omega_{t+1}^{(k)}}{\sum_{i=1}^{M} \omega_{t+1}^{(k)}}$ to obtain $\left\{x_{t+1}^{(k)}, k=1, \ldots, M\right\}$ and $\left\{\Theta_{t+1}^{(k)}, k=1, \ldots, M\right\}$. The posterior density $p\left(x_{t+1}, \Theta_{t+1} \mid D_{t+1}\right)$ can be approximated by $p\left(x_{t+1}, \mid D_{t+1}, \Theta_{t+1}\right)$. $p\left(\Theta_{t+1} \mid D_{t+1}\right)$ through these particles.

Repeating steps 1-3 iteratively until all the equity data information has been incorporated, the final posterior density approximation $p\left(x_{T}, \Theta_{T} \mid D_{T}\right)$ is our estimation.

\subsection{The model application}

\subsubsection{Credit risk measuring}

The most appealing reason for developing the SV structural model is its usage in credit risk measuring. Typically, the credit spread of a risky corporate bond over the corresponding risk-free interest rate, and the likelihood of a firm defaulting, are two commonly used indicators to evaluate the credit risk of private firms. Here we show how these credit risk indicators can be computed based on the SV structural model.

The credit spread of a risky corporate bond is defined as the premium required to compensate for the expected loss in the event of default, that is, $s_{t}=y_{t}-r$, where $y_{t}$ is the yield of the risky corporate bond, and $r$ is the risk-free interest rate. As discussed in Section 2.1, the risky debt can be priced by the difference between a default-free debt and a put option on the total asset value $V$ of the firm with the strike price of $F$ and the maturity date $T$. That is,

$$
B_{t}=F e^{-r(T-t)}-P_{t}^{H M}
$$

where $F$ is the face value of the zero coupon debt at the maturity time, and $P_{t}^{H M}$ is the price of a put option on the asset value $V$ with the strike price $T$ and the maturity date $T$. Take the Heston model described in Section 2.1 for example, once we estimate the model by using the observed equity values, the corresponding put option pricing formula based on Heston model can be used to compute $P_{t}^{H M}$ as ${ }^{7}$

$$
P_{t}^{H M}=F e^{-r(T-t)}\left(1-P_{2}\right)-S_{t}\left(1-P_{1}\right) .
$$

\footnotetext{
${ }^{7}$ We refer to Section 2.1 for the explicit expressions for $P_{1}$ and $P_{2}$.
} 
Then, the yield $y_{t}$ of the risky corporate bond can be derived from

$$
e^{-y_{t}(T-t)} F=B_{t}
$$

and the credit spread $s_{t}$ can be computed as

$$
s_{t}=-\frac{1}{T-t} \ln \left(1-\frac{P_{t}^{H M}}{F e^{-r(T-t)}}\right) .
$$

For the default probability of a firm, we resort to simulation to compute it, as its close form is unclear under the SV structural model. Once the parameter estimates of the model are obtained, we can simulate the evolution of the asset values a large number of times to approximate the distribution of firm's asset value at the debt maturity time $T$. Then the default probability can be computed by the probability of the asset value below the default barrier (the face value of the debt) at time $T$ over the simulated samples.

\subsubsection{Modeling microstructure noises}

The presence of market microstructure noise in equity prices has been well documented in the literature. When the observed equity prices are contaminated with microstructure noise, a fundamental estimation difficulty arises. Because the equity value is determined by both the underlying asset value and the trading noises, the previous one-to-one relationship between the observed equity value and the unobserved asset value no longer holds, and hence the data-transformed technique becomes infeasible. Duan and Fulop (2009) developed a simulation-based maximum likelihood (ML) method to estimate the Merton model with Gaussian i.i.d microstructure noises, and claimed that ignoring trading noises can lead to a significant overestimation of the asset return volatility. The SV structural model along with the particle filter estimation algorithm can be easily generalized to allow for trading noises in the observed equity prices.

Assuming a multiplicative error structure for the trading noises, the model is expressed as follows

$$
\ln E_{t}=\ln f\left(S_{t}, V_{t} ; \sigma, F, r, T-t\right)+\delta v_{t},
$$

where $v_{t}$ is i.i.d standard normal random variable, the option pricing function $f\left(S_{t}, V_{t} ; \sigma, F, r, T-\right.$ $t$ ) has been given in Section 2.1, and the asset value $S_{t}$ follows the Heston model as 
described in Section 2.1. Here, equation (11) with equation (1) and equation (2) constitutes a new state-space model with equation (11) being the measurement equation, and equation (1) and equation (2) being the transition equations. The estimation of this state-space system is also a non-linear and non-Gaussian filtering problem, and the only difference from the model we discussed in Section 2.1 is that the latent state vector now contains two variables, the asset value $S_{t}$ and the stochastic volatility $V_{t}$.

Our particle filter algorithm can be easily applied to estimate this system. Starting with the prior distribution $p\left(S_{t}, V_{t}, \Theta_{t} \mid D_{t}\right)$ represented by a set of particles $\left\{S_{t}^{(i)}, V_{t}^{(i)}, \Theta_{t}^{(i)}, i=\right.$ $1, \ldots, M\}$ with weights $\left\{\omega_{t}^{(i)}, i=1, \ldots, M\right\}$, where the parameter vector $\Theta$ denotes by $\Theta=\{\mu, \kappa, \theta, \xi, \rho, \delta\}$, the estimation procedure is summarized as:

- Step1 - Resample: Compute three mean points at time $t+1$ as

$$
\begin{gathered}
\mu_{V, t+1}^{(i)}=g\left(V_{t}^{(i)}\right)=E\left(V_{t+1} \mid V_{t}^{(i)}, \Theta_{t}^{(i)}\right), \\
\mu_{S, t+1}^{(i)}=g\left(S_{t}^{(i)}\right)=E\left(S_{t+1} \mid S_{t}^{(i)}, \mu_{V, t+1}^{(i)}, \Theta_{t}^{(i)}\right), \\
m_{t}^{(i)}=\alpha \Theta_{t}^{(i)}+(1-\alpha) \overline{\Theta_{t}}
\end{gathered}
$$

and sample a set of $M$ indexes $\{k, k=1, \ldots, M\}$ from $\{i, i=1, \ldots, M\}$ with weight

$$
\omega_{t+1}^{(i)} \propto \omega_{t}^{(i)} p\left(E_{t+1} \mid \mu_{S, t+1}^{(i)}, m_{t}^{(i)}\right)
$$

- Step2 - Prediction: Propagate $\left\{\Theta_{t}^{(k)}, k=1, \ldots, M\right\}$ to $\left\{\widetilde{\Theta}_{t+1}^{(k)}, k=1, \ldots, M\right\}$ by using the kernel density defined as

$$
\widetilde{\Theta}_{t+1}^{(k)} \sim N\left(. \mid m_{t}^{(k)}, h^{2} \Sigma_{t}\right)
$$

and propagate $\left\{S_{t}^{(k)}, V_{t}^{(k)}, k=1, \ldots, M\right\}$ to $\left\{\widetilde{S}_{t+1}^{(k)}, \widetilde{V}_{t+1}^{(k)}, k=1, \ldots, M\right\}$ via

$$
p\left(V_{t+1} \mid V_{t}^{(k)}, \widetilde{\Theta}_{t+1}^{(k)}\right)
$$

and

$$
p\left(S_{t+1} \mid S_{t}^{(k)}, \widetilde{V}_{t+1}^{(k)}, \widetilde{\Theta}_{t+1}^{(k)}\right)
$$


- Step 3 - Resample: Compute the corresponding weight as

$$
\omega_{t+1}^{(k)} \propto \frac{p\left(E_{t+1} \mid \widetilde{S}_{t+1}^{(k)}, \widetilde{\Theta}_{t+1}^{(k)}\right)}{p\left(E_{t+1} \mid \mu_{S, t+1}^{(k)}, m_{t}^{(k)}\right)},
$$

and resample $\left\{\widetilde{S}_{t+1}^{(k)}, \widetilde{V}_{t+1}^{(k)}, k=1, \ldots, M\right\}$ and $\left\{\widetilde{\Theta}_{t+1}^{(k)}, k=1, \ldots, M\right\}$ with weight $\pi_{t+1}^{(k)}=\frac{\omega_{t+1}^{(k)}}{\sum_{i=1}^{M} \omega_{t+1}^{(k)}}$ to obtain $\left\{S_{t+1}^{(k)}, V_{t+1}^{(k)}, k=1, \ldots, M\right\}$ and $\left\{\Theta_{t+1}^{(k)}, k=1, \ldots, M\right\}$. The posterior density $p\left(S_{t+1}, V_{t+1}, \Theta_{t+1} \mid D_{t+1}\right)$ can be approximated by $p\left(S_{t+1} \mid, V_{t+1}, \Theta_{t+1}, D_{t+1}\right)$. $p\left(V_{t+1} \mid \Theta_{t+1}, D_{t+1}\right) \cdot p\left(\Theta_{t+1} \mid D_{t+1}\right)$ through these particles.

Meanwhile, modeling the microstructure noise as an i.i.d normal variable is just a starting point. It is well known that the market microstructure effects are complex and can take many different forms. For example, as empirical facts suggest that the distribution of most financial variables have fact tails, Huang and Yu (2010) proposed to model the microstructure noise by a Student-t distribution. Moreover, the microstucture noise is likely to be correlated with the equity value. Note that these variations can be easily accommodated by the SV structural credit risk model, and the corresponding models can be easily estimated by the particle filter algorithm, as the algorithm is free of the distribution assumption on the model error term.

\section{Simulation study}

In this section, we conduct a simulation experiment to ascertain the finite sample performance of our data-transformed particle filter method for estimating the SV structural credit risk model. We firstly run a simulation study to investigate the finite sample performance of our transformed-data particle filter. We generate sample paths of four-year asset price observations for a firm from the Heston model (see equations (1) and (2)), and compute the corresponding equity values using the Heston option pricing formula (see equation (5)). We set $d t=1 / 250^{8}$ to reflect that the sample is simulated on a daily basis, and therefore we yield a sample of $1004(251 \times 4)$ asset and equity values. The initial maturity period of the firm's debt is set to 10 years, and gradually declines to 6 years at the end of the simulated sample. When estimating the model parameters,

\footnotetext{
${ }^{8}$ I follow Duan (2005) to set the number of the observations over a year as 250 rather than 365 , to account for holidays.
} 
we act as if we do not know the asset price values, and only utilize the information embedded in the observed equity values.

The parameter values we use in the simulation are consistent with the real data. For the baseline case, we take the median values of the 27 US firms in our empirical analysis, that is, $\mu=0.07, \kappa=0.2, \theta=0.144, \xi=0.08, \rho=-0.5$, and $F=0.5$. The end-of-sample pseudo-leverage ratio, $\frac{F}{V}$, is around $20 \%$. The risk-free interest rate $r$ is set to be 0.03 . We also vary the parameter values to investigate their effects on the performance of the method. The set of parameter values are changed to $\mu=0.1$, $\kappa=0.25, \theta=0.25, \xi=0.12, \rho=-0.8$, which are chosen based on the 90 percentile of the estimates obtained from the 27 US firms. We vary one parameter value at a time, and run the estimation by using 1000 particles in each case.

The prior distribution for the parameters' vector $\Theta$ we hypothesize here is consistent with Eraker et al. (2003). They are as follows: $\mu \sim N(0,0.1), \kappa \sim \operatorname{Beta}(0.25,0.2)$, $\theta \sim N(0,0.25), \xi^{2} \sim I G(0.144,0.05), \rho \sim U(-1,1)$, where, in particular, IG denotes the inverse Gamma distribution. We also performed a sensitivity analysis for these parameters. We considered different initial distributions for them, and found that all of these parameters were insensitive to the prior's choice.

For the baseline case, Figure 1 reports the sequential learning process for each parameter, including the evolution of the posterior mean together with the $2.5 \%$ and the 97.5\% posterior quantiles. Figure 2 displays the estimated latent volatility against the true volatility process. It is worth noting that our algorithm provides accurate estimates for all the parameters and the latent volatility process, as the posterior means of each parameter quickly converge to their true values, and the estimated volatility closely follows the true process. We replicate the above simulation 1000 times to eliminate the effect of the Monte Carlo error, and report (the average of) the 1000 parameter estimates in Table 1. Both median and mean values of all the parameter estimates are close to the true values with a relatively small sample of around 1000 observations, indicating the very good finite-sample behavior of our estimation algorithm.

The above analysis has abstained from trading noises. We take a further study to examine the performance of our estimation algorithm when the equity prices are contaminated by trading noises. As discussed in Section 2.3.2, the estimation becomes much more complex in this context due to the fact that the previous one-to-one relationship between the unobserved asset value and the observed equity price no longer exists. Like the latent stochastic volatility, the asset value is also a latent state variable which needs to be estimated. We take the above simulated equity price values from the 
baseline case, and add in a multiplicative error term $\delta \nu$ as the trading noises, where $\nu$ is a standard normal random variable. We firstly follow Duan and Fulop (2009) to set $\delta=0.004$, and change the value of this parameter to $\delta=0.016$ to look into its effect on the performance of our algorithm.

Figure 3 provides the estimated values of the unobserved asset and volatility processes when trading noises are present in equity prices. We also replicate the simulation by 1000 times, and report the (average) parameter estimation results in Table 2. The effect of trading noises does not fundamentally alter the quality of our estimation algorithm, as all the parameters are accurately estimated through the particle filter.

\section{Empirical analysis}

To implement our SV structural credit risk model on the real data, we conduct an empirical study to assess the credit risk of the 27 companies $^{9}$ that constitute the Dow Jones Industrial Index over the period of 2007-2012. Meanwhile, we will compare the ability of the Merton model and our SV structural model in terms of their average estimation errors of 5-year CDS spreads for the 27 companies. We use CDS spreads to test the performance of our model due to the fact that CDS spreads are a relatively pure pricing of default risk of the underlying entity, and the CDS contract is typically traded on standardized terms. Meanwhile, in the short run CDS spreads tend to respond efficiently to changes in credit conditions.

\subsection{Data Description}

Our data sample consists of daily 5-year CDS spreads of the 27 companies of the Dow Jones Industrial Index, and all required balance sheet information for computing model implied credit spreads of these firms, over the period of years 2007-2012. We choose 5-year maturity CDS spreads as the 5-year maturity CDS are the most liquid contracts traded in the US credit market, and the data of the CDS spreads are taken from Datastream. Company names and main statistics of these CDS spreads are summarized in Table 4. The equity values of these firms are computed as the product of the closing price of equity and number of shares outstanding obtained from the CRSP database. The initial maturity of debt is set to 5 years. We take the book value of liabilities

\footnotetext{
${ }^{9}$ Three component companies are left out because either the data do not cover the whole sample period or the CDS spreads are constant during long periods of time.
} 
of these companies at the year end of 2006-2011 and compound it for 5 years at the risk-free interest rate. The resulting value is our proxy for the face value of the debt in our model. The risk-free interest rate is the 1-year Treasury constant maturity rate obtained from the US Federal Reserve. To consider both in-sample fit and out-of-sample forecast performance, we split the sample into two periods. The first 885 observations from years 2007-2010 of the sample are used for model estimation, while the last 445 observations in the last two years are used for out-of-sample forecast analysis. After the initial estimation period, a recursive estimation scheme is employed to obtain parameter estimates upon which the sequence of one-step ahead forecasts of CDS spreads are generated. We run the estimation using the 5000-particle filter.

\subsection{Empirical Results}

We firstly investigate the in-sample fit of the SV structural model and the Merton model. We estimate the two models based on the first 885 observations of the insample period ${ }^{10}$, then use the in-sample parameter estimates, together with the risk-free interest rate and the face value of the debt at each time point (of the in-sample period), to produce a sequences of corporate bond credit spread estimations that relate to the in-sample period. To illustrate the importance of incorporating stochastic volatility into credit risk modeling, we employ 5-year CDS spreads as a proxy of the real credit risk of these firms, and use root mean square error (RMSE) and mean absolute error (MAE) of the credit risk estimation to compare the performance of the two models. The RMSE of credit risk estimation for each sample firm has the standard definition as

$$
R M S E=\sqrt{E\left(R S_{i, t}-I S_{i, t}\right)^{2}}
$$

and MAE is then estimated for each sample firm as

$$
M A E=E\left(\left|R S_{i, t}-I S_{i, t}\right|\right)
$$

where $R S_{i, t}$ is the real 5-year CDS spread for firm $\mathrm{i}$ at the end of day $\mathrm{t}$, and $I S_{i, t}$ is the model implied credit spread for firm $\mathrm{i}$ at the end of day t. The $I S_{i t}$ from the SV structural model are calculated according to equation (10), and the ones from the Merton model refer to Duan (1994).

\footnotetext{
${ }^{10}$ The SV structural model is estimated by our transformed-data particle filter algorithm, and the Merton model is estimated by the transformed-data MLE method developed in Duan (1994).
} 
Table 5 reports the SV structural model estimation results for all Dow Jones firms based on the first 885 observations. Names are given in the first column. The rest of the columns contain the posterior mean of the model parameter estimates along with the 95\% confidence bounds. The $95 \%$ confidence intervals are very small, implying these parameters have been fairly accurately estimated. The summary of results from these firms are reported in Table 6 . The estimated long-run asset volatilities $\theta$ are stated per annum for each firm and are consistent with the range that is expected of their values, and all the other parameters are presented on a daily basis. More importantly, the parameters $\xi$ that indicate the volatility of the asset volatility are statistically significant across all the firms at the $5 \%$ significance level, which confirms that the asset volatilities of these firms are a stochastic process rather than a constant.

Table 6 summarizes the in-sample RMSE and MAE fit of both models for each sample firm, and the mean, median and interquartile of RMSE and MAE fit of all these firms. Our SV structural model always achieves a lower RMSE and MAE than the Merton model for all the sample firms. The RMSE and MAE of credit spread estimation from the Merton model are approximately 115.30bps and 129.67bps on average respectively, while our SV structural model largely reduces the estimation error to 100.45bps and $113.33 \mathrm{bps}$ respectively.

A model's out-of-sample performance seems more important in evaluating a model's ability. A more richly parameterized model is normally expected to perform better insample than a more sparsely parameterized alternative model, but this may not be the case for out-of-sample performance. The reason is that models with extra parameters may be penalized in an out-of-sample analysis because of the difficulty in identifying those extra parameters, given the limited sample size of available data. Here, we use the last two years as the out-of-sample period to compare the performance of the two models. The rest of the out-of-sample analysis is as follows. We firstly use the in-sample parameter estimates of the two models based on the first 885 observations to generate one-step-ahead forecasts of the corporate bond credit spread in next period. Then we re-estimate these parameters upon the new observations available to generate sequences of one-step-ahead forecasts of the corporate bond credit spread that relate to the outof-sample forecast period. Finally, we compute the corresponding RMSE and MAE to gauge the out-of-sample performance of the two models. The out-of-sample results are presented in Table 7. Again, it is clear that the SV structural model compares favorably with the Merton model in out-of-sample forecasting: the RMSEs and MAE from the SV structural model in every single firm are less than those from the Merton model. 
Meanwhile, the mean RMSE and MAE in the SV structural model are 102.82bps and 114.10bps, down from 116.06bps and 126.32bps in the Merton model.

\section{Conclusion}

We have developed a SV structural credit risk model along with a transformed-data particle filter estimation method in order to improve the performance of the Merton model in credit risk measuring. The simulation study verifies the good performance of our model estimation algorithm, and the empirical analysis ascertains the importance of recognizing the stochastic property of the asset return volatility in the credit risk modeling, by showing that the SV structural model fits the actual CDS spread much better than the Merton model. Although our methodological development is presented specifically for the Heston model, the method developed here can be very easily adapted to other stochastic volatility structural credit risk models. This is in a way similar to the fact that the particle filter can be applied to general stochastic volatility models. Furthermore, in this paper, asset returns and volatility are assumed to follow a stochastic process without jumps. It is also straightforward to allow for jumps in this model, and our estimation methods can be easily applied too. In conclusion, a stochastic volatility structural credit risk model has been developed to measure the credit risk of a firm or a market in which the asset return volatility is not constant. 


\section{References}

[1] Bakshi, G., Cao, C., Chen, Z., 2000. Do call prices and the underlying stock always move in the same direction? Review of Financial Studies 13, 549-584.-0.6895(0.0056)

[2] Eom, Y.H., Helwege, J., Huang, J.Z., 2004. Structural Models of Corporate Bond Pricing: An Empirical Analysis. Review of Financial Studies 17, 499544.

[3] Eraker, B., Johannes, M., Polson, N., 2003. The impact of jumps in volatility and returns. Journal of Finance 58, 1269-1300.

[4] Duan, J.-C., 1994. Maximum likelihood estimation using price data of the derivative contract. Mathematical Finance 4, 155-167.

[4] Duan, J.-C., 2000. Correction: Maximum likelihood estimation using price data of the derivative contract. Mathematical Finance 10, 461-462.

[5] Duan, J.-C., Fulop, A., 2009. Estimating the structural credit risk model when equity prices are contaminated by trading noises. Journal of Econometrics 150 (2), 288-296.

[6] Huang, S.J., Yu, J., 2010. Bayesian analysis of structural credit risk models with microstructure noises. Journal of Economic Dynamics and Control 34 (11), 2259-2272.

[7] Jones, E.P., Mason, S.P., Rosenfeld, E., 1984. Contingent Claims Analysis of Corporate Capital Structures: An Empirical Investigation. Journal of Finance 39, 611-625.

[8] Liu, J.S., Chen, R., 1998. Sequential Monte Carlo methods for dynamic systems. J. Amer. Statist. Assoc. [This journal title shouldn't be abbreviated.] $93,1032-1044$.

[9] Liu, J., West, M., 2001. Combined parameters and state estimation in simulation-based filtering. In: Doucet, A., de Freitas, N., Gordon, N. (Eds.), Sequential Monte Carlo Methods in Practice. Springer, New York, 197-223. 
[10] Merton, R.C., 1974. On the pricing of corporate debt: the risk structure of interest rates. Journal of Finance 29, 449-70.

[11] Ogden, J., 1987. Determinants of Ratings and Yields on Corporate Bonds: Tests of the Contingent Claims Model. Journal of Financial Research 10, 329-339.

[12] Pitt, M.K., Shephard, N., 1999. Filtering Via Simulation: Auxiliary Particle Filters. Journal of the American Statistical Association 94, 590-591.

[13] Stein, J.C., 1989. Overreactions in the options market. Journal of Finance 44, 1011-1023.

[14] Tarashev, N., 2005. Theoretical predictors of default: Lessons from firmlevel data. BIS Quarterly Review, September. 


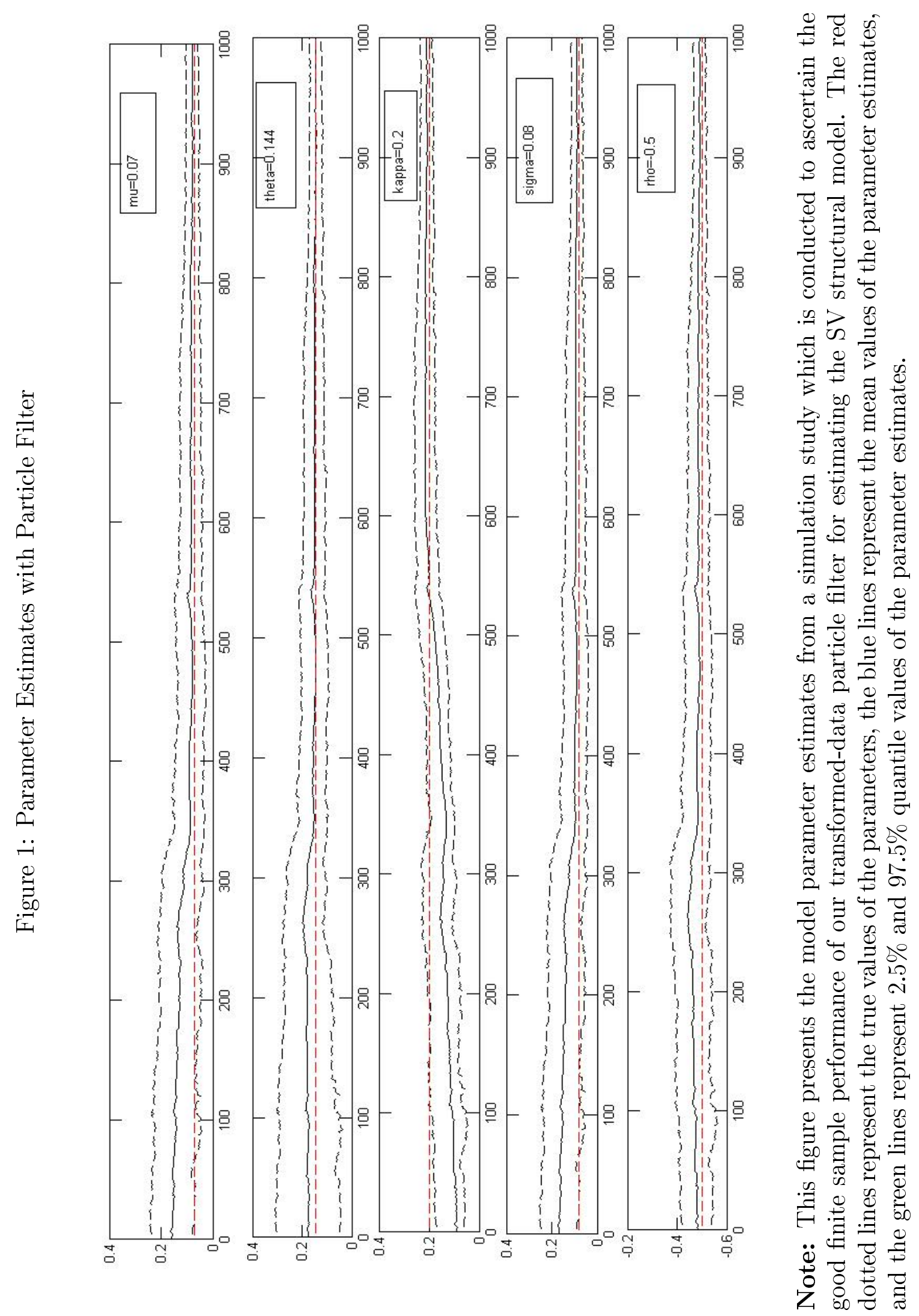




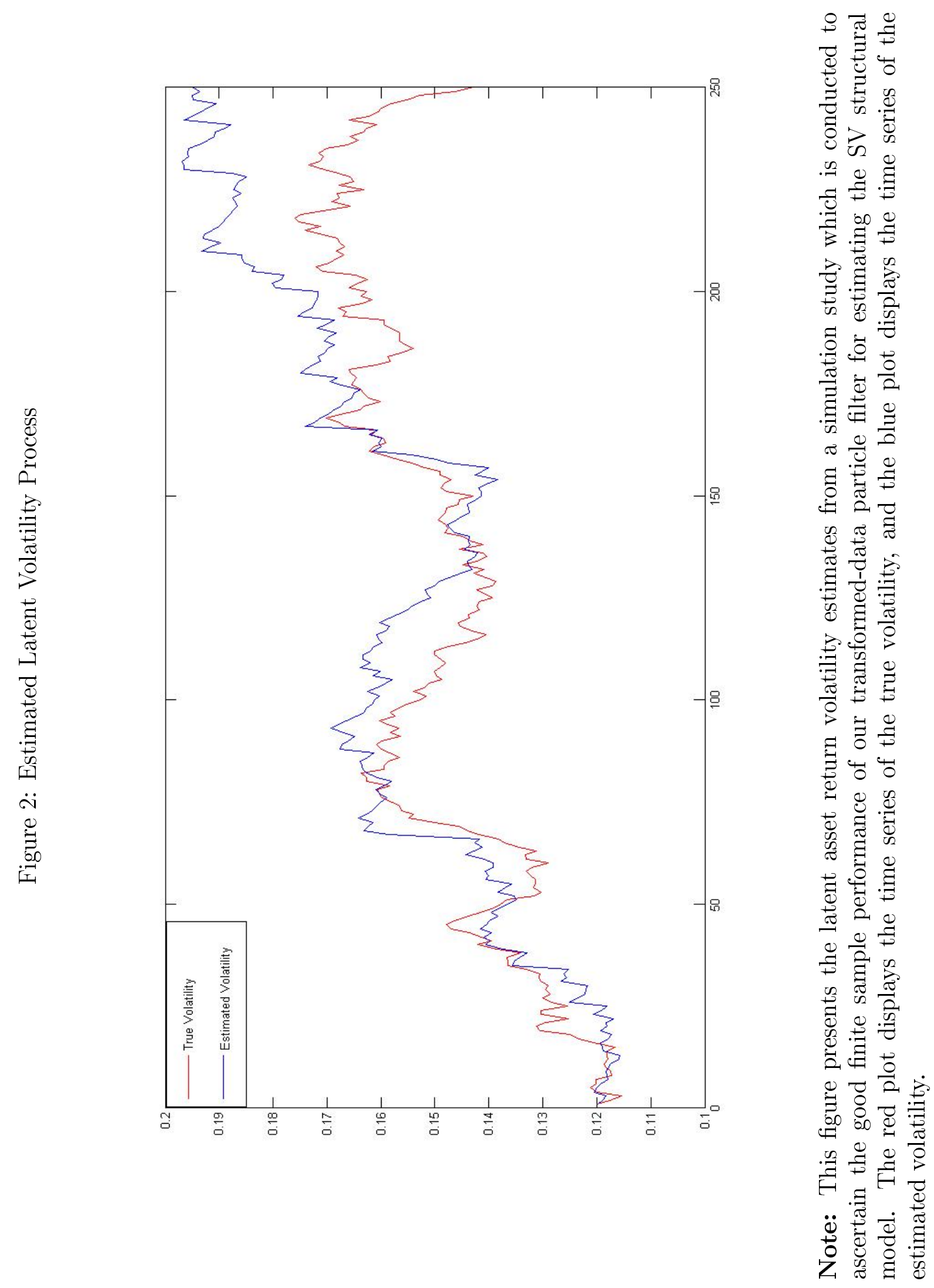




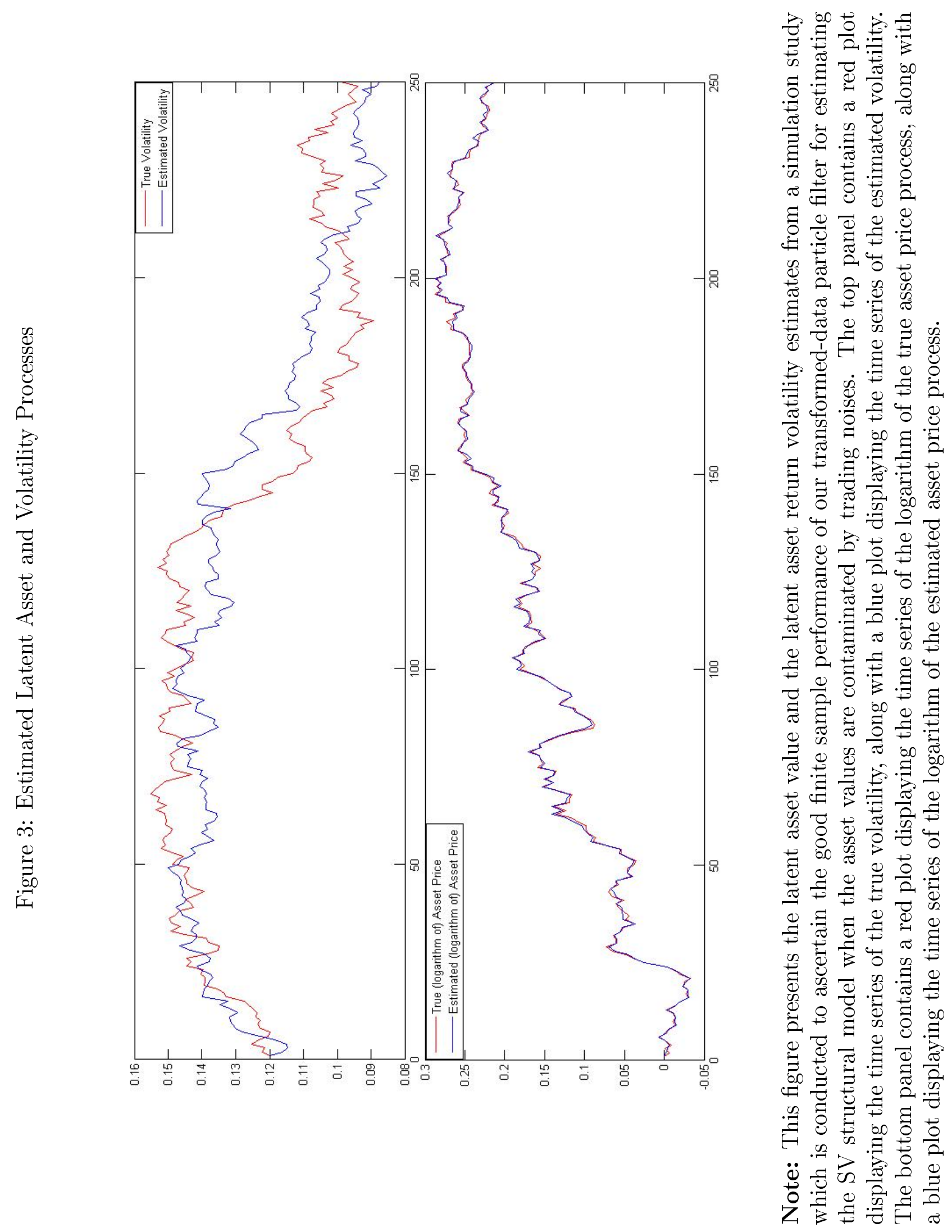


Table 1: Parameter Estimation Results

\begin{tabular}{cccccc}
\hline \multicolumn{7}{c}{ Estimated Parameters } \\
\hline Mean & $\mu=0.07$ & $\kappa=0.2$ & $\theta=0.144$ & $\xi=0.08$ & $\rho=-0.5$ \\
\cline { 2 - 6 } Median & 0.0711 & 0.2078 & 0.1442 & 0.0810 & -0.5100 \\
$2.5 \%$ percentile & 0.0709 & 0.2081 & 0.1443 & 0.0809 & -0.5102 \\
$25 \%$ percentile & 0.0692 & 0.1967 & 0.1425 & 0.0786 & -0.4876 \\
$75 \%$ percentile & 0.0756 & 0.2212 & 0.1476 & 0.8123 & -0.5182 \\
$97.5 \%$ percentile & 0.0789 & 0.2309 & 0.1498 & 0.8221 & 0.5234 \\
\hline & \multicolumn{5}{c}{ Estimated Parameters } \\
\hline Mean & $\mu=0.1$ & $\kappa=0.25$ & $\theta=0.25$ & $\xi=0.12$ & $\rho=-0.8$ \\
\hline Median & 0.1002 & 0.2516 & 0.2504 & 0.1203 & -0.8097 \\
$2.5 \%$ percentile & 0.0969 & 0.2504 & 0.2505 & 0.1205 & -0.8101 \\
$25 \%$ percentile & 0.0972 & 0.21972 & 0.2478 & 0.1187 & -0.7965 \\
$75 \%$ percentile & 0.1063 & 0.2715 & 0.2490 & 0.1198 & -0.7997 \\
$97.5 \%$ percentile & 0.1109 & 0.2912 & 0.2565 & 0.1221 & -0.8210 \\
\hline
\end{tabular}

Note: We conduct a simulation experiment to ascertain the finite sample performance of our data-transformed particle filter method for estimating the SV structural credit risk model. We generate sample paths of four-year asset price observations for a firm from the Heston model, and compute the corresponding equity values using the Heston option pricing formula. We set $d t=1 / 250$ to reflect that the sample is simulated on a daily basis, and therefore we yield a sample of 251 asset and equity values. The initial maturity period of the firm's debt is set to 10 years, and gradually declines to 6 years at the end of the simulated sample. When estimating the model parameters, we act as if we do not know the asset price values, and only utilize the information embedded in the observed equity values. We only report the parameter estimates for the two cases where $\{\mu=0.07, \kappa=0.2, \theta=0.144, \sigma=0.08, \rho=-0.5\}$ and $\{\mu=0.1, \kappa=0.25, \theta=0.25, \sigma=0.12, \rho=-0.8\}$ in this table. The estimation results for other cases are available upon request. 
Table 2: Parameter Estimation ResultsIncorporating Trading Noises

\begin{tabular}{ccccccc}
\hline \multicolumn{7}{c}{ Estimated Parameters } \\
\hline \multirow{2}{*}{ Mean } & $\mu=0.07$ & $\kappa=0.20$ & $\theta=0.144$ & $\xi=0.08$ & $\rho=-0.5$ & $\delta=0.004$ \\
\cline { 2 - 7 } Median & 0.0708 & 0.2081 & 0.1444 & 0.0805 & -0.5103 & 0.0041 \\
$2.5 \%$ percentile & 0.0712 & 0.2082 & 0.1447 & 0.0812 & -0.5105 & 0.0043 \\
$25 \%$ percentile & 0.0687 & 0.1971 & 0.1431 & 0.0765 & -0.4884 & 0.0038 \\
$75 \%$ percentile & 0.0749 & 0.2209 & 0.1440 & 0.0789 & -0.4976 & 0.0039 \\
$97.5 \%$ percentile & 0.0785 & 0.2312 & 0.1484 & 0.8132 & -0.5190 & 0.0045 \\
\hline \multicolumn{7}{c}{ Estimated Parameters } \\
\hline & $\mu=0.07$ & $\kappa=0.25$ & $\theta=0.144$ & $\xi=0.08$ & $\rho=-0.5$ & $\delta=0.016$ \\
\hline Mean & 0.0712 & 0.2584 & 0.1448 & 0.0810 & -0.5109 & 0.0167 \\
Median & 0.0713 & 0.2585 & 0.1450 & 0.0813 & -0.5112 & 0.0168 \\
$2.5 \%$ percentile & 0.0672 & 0.1873 & 0.1439 & 0.0770 & -0.4890 & 0.0161 \\
$25 \%$ percentile & 0.0693 & 0.2197 & 0.1448 & 0.0793 & -0.4982 & 0.0163 \\
$75 \%$ percentile & 0.0751 & 0.2725 & 0.1482 & 0.8140 & -0.5193 & 0.0169 \\
$97.5 \%$ percentile & 0.0792 & 0.2720 & 0.1491 & 0.8257 & 0.5225 & 0.0170 \\
\hline
\end{tabular}

Note: All the simulation set-ups are the same as the one in Table 1. The extra parameter involved here is the one for trading noise. We firstly follow Duan and Fulop (2009) to set $\delta=0.004$, and change the value of this parameter to $\delta=0.016$ to look into its effect on the performance of our algorithm. 


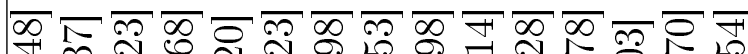
10.

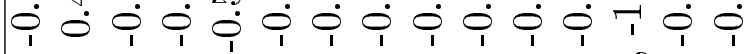

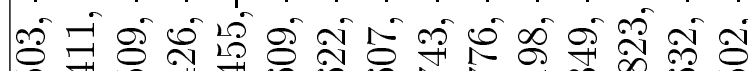

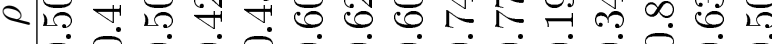

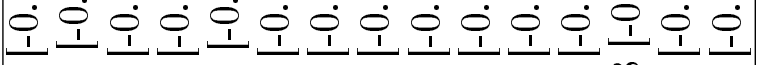

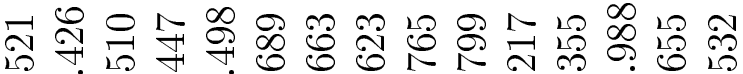

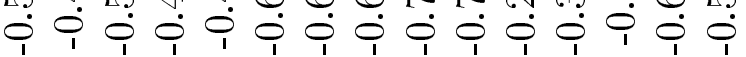

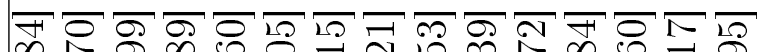

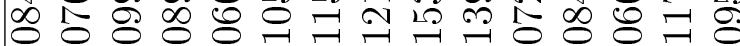

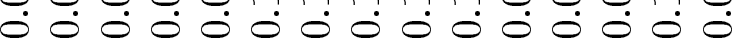
แก่

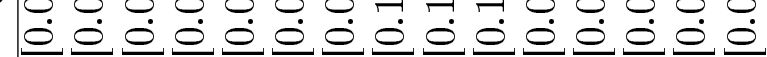

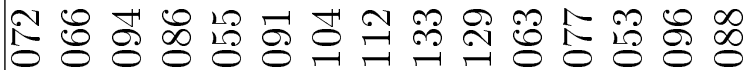

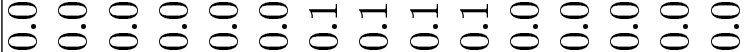

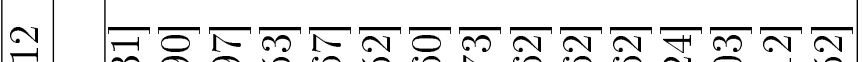

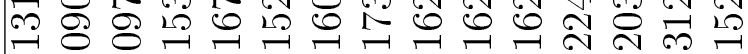

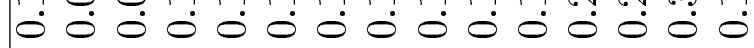
- 20 की Fo. 든

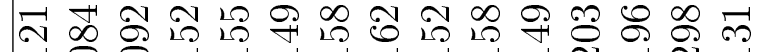

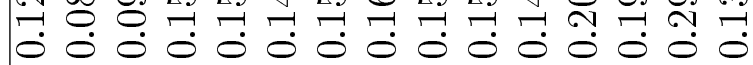

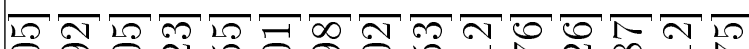

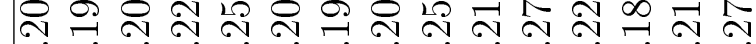

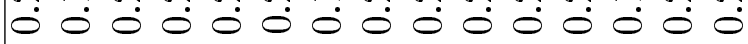

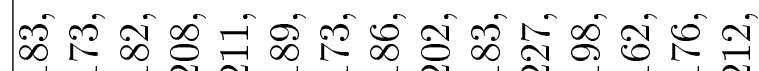

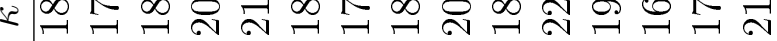

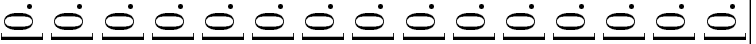

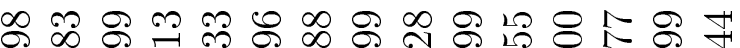
:

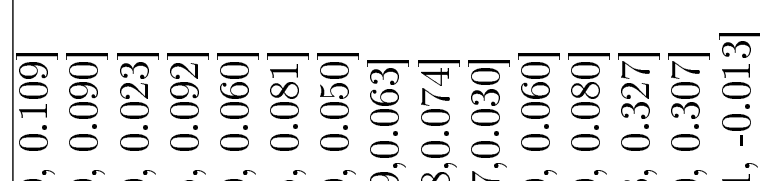

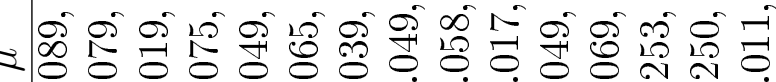

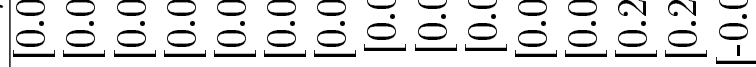
的

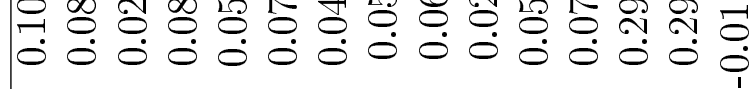

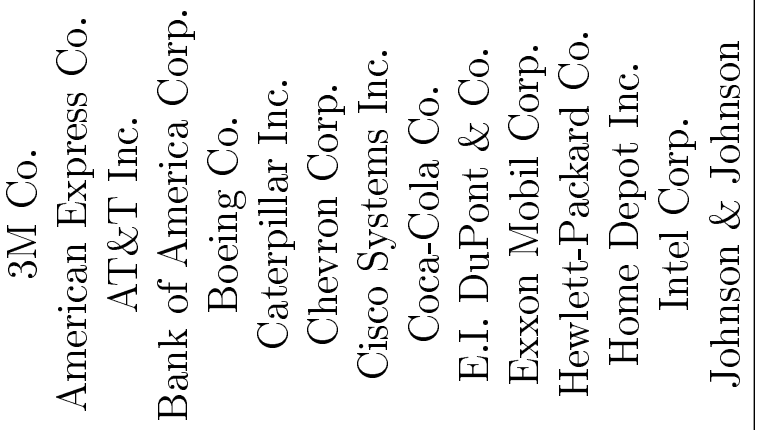

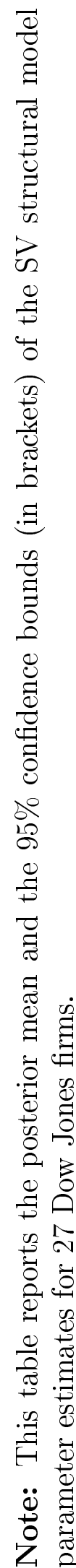




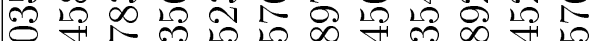

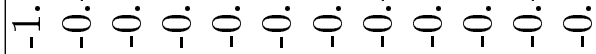
के

Q

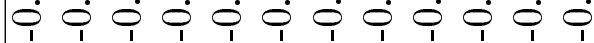
$\infty$ ॠ

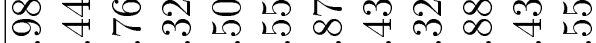

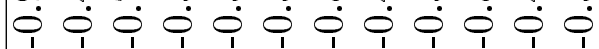

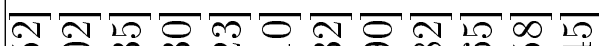

ㄱำ

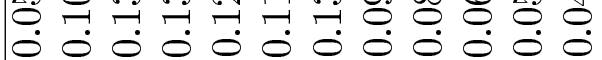

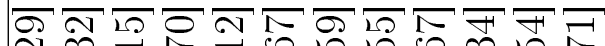

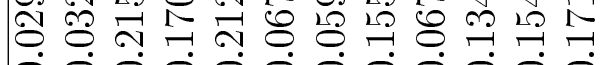

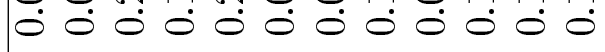

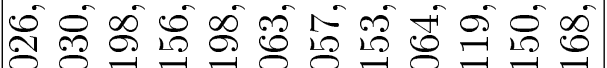
$\dot{0} \dot{\varrho} \dot{\varrho} \dot{\varrho} \dot{\varrho} \dot{\varrho} \dot{\varrho} \dot{\varrho} \dot{\varrho}$

艎

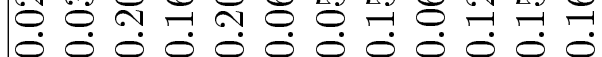

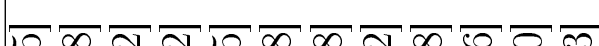

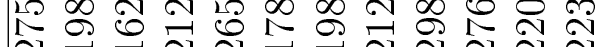

ๆ ઍ

ฟ $\dot{0} \dot{0} \dot{0} \dot{0} \dot{0} \dot{0} \dot{0} \dot{0} \dot{0} \dot{0}$

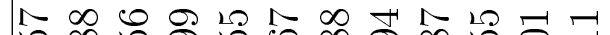
๙ิ

它

ㄷㅇㅇㅇㅇㅇㅇㅇㅇㅎㅇㅇㅠ

F

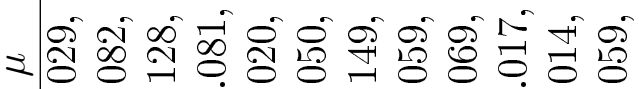

$\dot{0} \dot{0} \dot{0} \dot{0} \dot{0} \dot{0} \dot{0} \dot{0} \dot{0} \dot{0} \dot{0} \dot{0}$

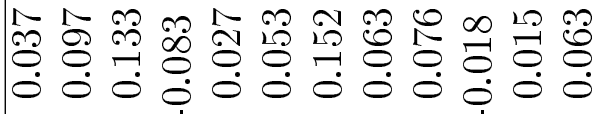

T)

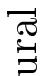

晜

s

$\stackrel{0}{\varpi}$

茨

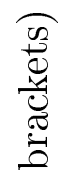

$\Xi$

告

ن.

응

身

ซี

สี

E

$\begin{array}{ll}0 & 0 \\ 0 & 0 \\ 0 & 0 \\ 0 & 0 \\ 0 & 0 \\ 0 & 0 \\ 0 & 0\end{array}$

$\stackrel{\circ}{\square}$

次

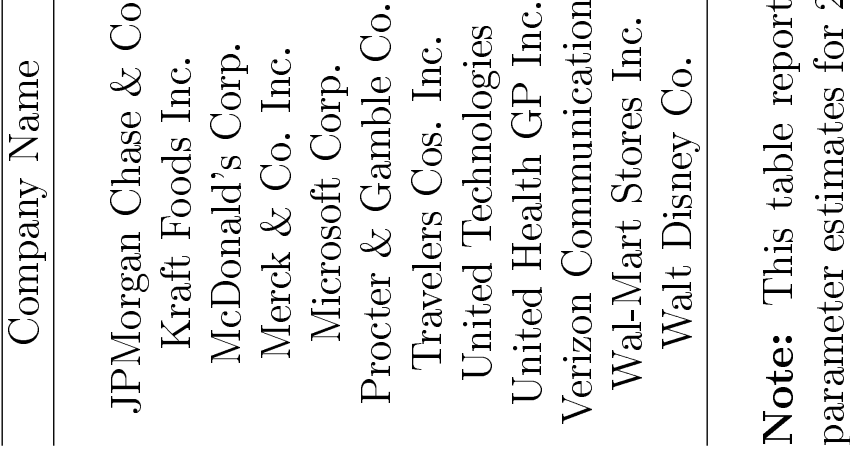


Table 4: Summary of the SV Structural Model Estimation for Dow Jones Companies

\begin{tabular}{cccccc}
\hline Company Name & \multicolumn{5}{c}{ Jan 2007-Dec 2012} \\
\hline & $\mu$ & $\kappa$ & $\theta$ & $\xi$ & $\rho$ \\
\cline { 2 - 6 } Mean & 0.0719 & 0.2104 & 0.1405 & 0.0890 & -0.5850 \\
Median & 0.0631 & 0.1993 & 0.1522 & 0.0887 & -0.5323 \\
10th Percentile & -0.0128 & 0.1775 & 0.0587 & 0.0534 & -0.3230 \\
90th Percentile & 0.1522 & 0.2554 & 0.2033 & 0.1298 & -0.8870 \\
Min & -0.0831 & 0.1567 & 0.0287 & 0.0431 & -0.9887 \\
Max & 0.2946 & 0.2877 & 0.2988 & 0.1334 & -0.2178 \\
\hline
\end{tabular}

Note: This table reports the summary statistics of the SV structural model parameter estimates for 27 Dow Jones firms. 
Table 5: Summary Statistics of the 5-year CDS Spreads for the Constituent Companies of the Dow Jones Industrial Index

\begin{tabular}{|c|c|c|c|c|}
\hline Company Name & \multicolumn{4}{|c|}{ Jan 2007-Dec 2012} \\
\hline & Mean & Std. & Max & Min \\
\hline 3M Co. & 45.652 & 21.471 & 125.000 & 17.000 \\
\hline American Express Co. & 171.211 & 140.336 & 738.850 & 65.201 \\
\hline AT\&T Inc. & 91.729 & 30.376 & 232.500 & 54.160 \\
\hline Bank of America Corp. & 180.746 & 88.432 & 500.480 & 51.664 \\
\hline Boeing Co. & 100.316 & 56.879 & 295.000 & 30.500 \\
\hline Caterpillar Inc. & 101.973 & 65.316 & 452.500 & 31.500 \\
\hline Chevron Corp. & 48.242 & 20.941 & 135.000 & 16.000 \\
\hline Cisco Systems Inc. & 68.141 & 26.015 & 172.500 & 27.310 \\
\hline Coca-Cola Co. & 44.582 & 14.907 & 95.000 & 21.500 \\
\hline E.I. DuPont \& Co. & 68.831 & 20.941 & 135.000 & 16.000 \\
\hline Exxon Mobil Corp. & 35.589 & 17.093 & 117.150 & 19.630 \\
\hline Hewlett-Packard Co. & 61.739 & 33.586 & 158.500 & 21.200 \\
\hline Home Depot Inc. & 101.921 & 64.374 & 347.500 & 41.220 \\
\hline Intel Corp. & 58.655 & 24.388 & 88.500 & 26.175 \\
\hline Johnson \& Johnson & 38.682 & 12.557 & 75.000 & 10.000 \\
\hline JPMorgan Chase \& Co. & 103.614 & 36.673 & 310.000 & 45.160 \\
\hline Kraft Foods Inc. & 72.391 & 20.256 & 164.500 & 30.120 \\
\hline McDonald's Corp. & 38.005 & 11.098 & 75.000 & 20.140 \\
\hline Merck \& Co. Inc. & 59.405 & 9.686 & 79.154 & 38.201 \\
\hline Microsoft Corp. & 33.131 & 10.414 & 95.000 & 10.000 \\
\hline Procter \& Gamble Co. & 55.995 & 26.712 & 151.000 & 29.500 \\
\hline Travelers Cos. Inc. & 86.042 & 25.383 & 158.270 & 25.000 \\
\hline United Technologies & 59.726 & 26.765 & 147.500 & 25.000 \\
\hline United Health GP Inc. & 144.996 & 67.260 & 390.000 & 47.500 \\
\hline Verizon Communications & 79.517 & 24.103 & 175.000 & 40.180 \\
\hline Wal-Mart Stores Inc. & 52.664 & 19.943 & 137.500 & 25.000 \\
\hline Walt Disney Co. & 48.348 & 17.633 & 130.000 & 23.540 \\
\hline Average & 75.994 & 34.575 & 210.422 & 29.941 \\
\hline
\end{tabular}

Note: This table reports the summary statistics of the 5-year CDS spreads over years 2007-2012 for 27 Dow Jones firms. 
Table 6: In-Sample Performance of SV Structural Model and Merton Model for Estimating 5-year CDS Spreads

\begin{tabular}{ccccc}
\hline Company Name & \multicolumn{2}{c}{ Merton Model } & \multicolumn{2}{c}{ SV Structural Model } \\
\cline { 2 - 5 } 3M Co. & 115.46 & 137.89 & 95.34 & 112.24 \\
\cline { 2 - 5 } RMSE(bps) & MAE(bps) & RMSE(bps) & MAE(bps) \\
American Express Co. & 119.34 & 138.77 & 90.78 & 119.74 \\
AT\&T Inc. & 120.76 & 135.67 & 110.77 & 127.82 \\
Bank of America Corp. & 121.67 & 148.99 & 114.89 & 129.21 \\
Boeing Co. & 115.34 & 136.01 & 108.76 & 125.22 \\
Caterpillar Inc. & 111.40 & 143.99 & 101.21 & 129.87 \\
Chevron Corp. & 113.65 & 141.22 & 96.23 & 128.87 \\
Cisco Systems Inc. & 115.96 & 144.87 & 97.98 & 123.01 \\
Coca-Cola Co. & 122.33 & 149.75 & 109.37 & 121.87 \\
E.I. DuPont \& Co. & 120.88 & 150.11 & 108.90 & 124.29 \\
Exxon Mobil Corp. & 115.78 & 136.16 & 94.21 & 114.76 \\
Hewlett-Packard Co. & 110.89 & 132.45 & 97.21 & 118.92 \\
Home Depot Inc. & 116.79 & 131.88 & 96.91 & 116.23 \\
Intel Corp. & 118.92 & 145.87 & 94.49 & 124.21 \\
Johnson \& Johnson & 113.22 & 130.85 & 95.82 & 118.13 \\
JPMorgan Chase \& Co. & 114.56 & 137.64 & 96.90 & 126.32 \\
Kraft Foods Inc. & 113.44 & 115.22 & 101.25 & 104.85 \\
McDonald's Corp. & 115.67 & 113.87 & 109.20 & 105.85 \\
Merck \& Co. Inc. & 111.86 & 110.89 & 97.69 & 99.25 \\
Microsoft Corp. & 110.82 & 119.87 & 96.25 & 97.31 \\
Procter \& Gamble Co. & 112.49 & 114.75 & 102.94 & 101.32 \\
Travelers Cos. Inc. & 113.22 & 112.84 & 97.30 & 98.93 \\
United Technologies & 115.68 & 111.90 & 104.21 & 94.45 \\
United Health GP Inc. & 116.72 & 112.39 & 103.09 & 102.36 \\
Verizon Communications & 115.04 & 117.88 & 96.93 & 99.19 \\
Wal-Mart Stores Inc. & 110.64 & 115.45 & 95.29 & 96.18 \\
Walt Disney Co. & 110.45 & 113.85 & 98.27 & 99.41 \\
\hline Mean & 115.30 & 129.67 & 100.45 & 113.33 \\
Median & 115.34 & 132.45 & 97.69 & 116.23 \\
10th percentile & 110.82 & 112.39 & 94.49 & 97.31 \\
90th percentile & 120.88 & 148.99 & 109.37 & 128.87 \\
\hline & & & &
\end{tabular}

Note: This table reports the in-sample RMSEs of the SV structural model and the Merton model for estimating the 5-year CDS spreads. 
Table 7: Out-of-Sample Performance of SV Structural Model and Merton Model for Estimating 5-year CDS Spreads

\begin{tabular}{ccccc}
\hline Company Name & \multicolumn{2}{c}{ Merton Model } & \multicolumn{2}{c}{ SV Structural Model } \\
\cline { 2 - 5 } 3M Co. & 119.43 & 136.79 & 103.34 & 121.24 \\
\cline { 2 - 5 } RMSE(bps) & MAE(bps) & RMSE(bps) & MAE(bps) \\
American Express Co. & 120.56 & 134.67 & 101.78 & 109.74 \\
AT\&T Inc. & 119.83 & 133.79 & 104.77 & 121.82 \\
Bank of America Corp. & 122.77 & 145.87 & 102.89 & 129.21 \\
Boeing Co. & 119.04 & 137.24 & 101.76 & 115.22 \\
Caterpillar Inc. & 115.37 & 137.96 & 103.21 & 129.87 \\
Chevron Corp. & 107.82 & 139.42 & 106.23 & 128.87 \\
Cisco Systems Inc. & 119.86 & 142.77 & 107.98 & 123.01 \\
Coca-Cola Co. & 121.43 & 139.65 & 109.37 & 130.87 \\
E.I. DuPont \& Co. & 121.68 & 143.21 & 104.90 & 139.29 \\
Exxon Mobil Corp. & 119.28 & 129.46 & 100.21 & 111.76 \\
Hewlett-Packard Co. & 109.79 & 131.28 & 103.21 & 125.92 \\
Home Depot Inc. & 118.92 & 130.78 & 110.91 & 118.23 \\
Intel Corp. & 112.38 & 135.79 & 96.49 & 121.21 \\
Johnson \& Johnson & 113.28 & 132.57 & 105.82 & 128.13 \\
JPMorgan Chase \& Co. & 121.63 & 135.04 & 110.90 & 128.32 \\
Kraft Foods Inc. & 118.74 & 105.82 & 97.25 & 91.85 \\
McDonald's Corp. & 117.71 & 115.75 & 109.20 & 99.85 \\
Merck \& Co. Inc. & 112.34 & 108.79 & 97.69 & 95.25 \\
Microsoft Corp. & 107.26 & 118.73 & 96.25 & 97.31 \\
Procter \& Gamble Co. & 113.59 & 113.29 & 98.94 & 100.32 \\
Travelers Cos. Inc. & 112.21 & 117.43 & 107.30 & 108.93 \\
United Technologies & 118.29 & 113.78 & 104.21 & 105.45 \\
United Health GP Inc. & 115.23 & 113.47 & 107.09 & 108.36 \\
Verizon Communications & 113.24 & 107.32 & 96.93 & 99.19 \\
Wal-Mart Stores Inc. & 111.46 & 106.52 & 95.29 & 96.18 \\
Walt Disney Co. & 110.55 & 103.52 & 92.27 & 95.41 \\
\hline Mean & 116.06 & 126.32 & 102.82 & 114.10 \\
Median & 117.71 & 131.28 & 103.21 & 115.22 \\
10th percentile & 109.79 & 106.52 & 96.25 & 95.41 \\
90th percentile & 121.63 & 142.77 & 109.37 & 129.87 \\
\hline
\end{tabular}

Note: This table reports the out-of-sample RMSEs of the SV structural model and the Merton model for estimating the 5-year CDS spreads. 